\title{
The type of shared activity shapes caregiver and infant communication
}

\author{
Daniel Puccini ${ }^{\mathrm{a}}$, Mireille Hassemer ${ }^{\mathrm{a}}$, Dorothé Salomo ${ }^{\mathrm{a}}$, \\ and Ulf Liszkowski ${ }^{\mathrm{a}, \mathrm{b}}$ \\ ${ }^{a}$ Max Planck Research Group Communication Before Language, Max \\ Planck Institute for Psycholinguistics, Nijmegen, The Netherlands. / \\ bonders Institute for Brain, Cognition and Behaviour, Radboud University, \\ Nijmegen, The Netherlands.
}

\begin{abstract}
For the beginning language learner, communicative input is not based on linguistic codes alone. This study investigated two extralinguistic factors which are important for infants' language development: the type of ongoing shared activity and non-verbal, deictic gestures. The natural interactions of 39 caregivers and their 12-month-old infants were recorded in two semi-natural contexts: a free play situation based on action and manipulation of objects, and a situation based on regard of objects, broadly analogous to an exhibit. Results show that the type of shared activity structures both caregivers' language usage and caregivers' and infants' gesture usage. Further, there is a specific pattern with regard to how caregivers integrate speech with particular deictic gesture types. The findings demonstrate a pervasive influence of shared activities on human communication, even before language has emerged. The type of shared activity and caregivers' systematic integration of specific forms of deictic gestures with language provide infants with a multimodal scaffold for a usage-based acquisition of language.
\end{abstract}

Keywords: caregiver-infant communication, context, gesture, multimodal, speech-gesture combinations

Research investigating children's language input typically focuses on linguistic factors such as the semantics, syntax, or phonology of caregivers' speech. However, according to social-pragmatic theories, children's language acquisition is also heavily dependent on other, non-linguistic factors of communication (Baldwin, 1995; Bruner, 1975, 1981, 1983; Tomasello, 2003). Two extralinguistic factors that play a major role in children's acquisition of language are: (i) mutual engagement in shared activities and (ii) non-verbal, deictic gestures. 
Shared activities facilitate children's language learning on a micro and a macro level (Tomasello \& Farrar, 1986). On the micro level, shared activities enable infants to determine the referent of a word through a shared focus of attention (Tomasello \& Farrar, 1986; Baldwin, 1991). On the macro level, shared activities enable infants to learn about the different functions for which language is used (Nelson, 1981). Bruner (1983) argued that familiar, conventionalized routines in children's daily lives serve as 'formats' within which language becomes meaningful. According to Bruner, conventionalized routines allow infants to limit the amount of possible interpretations of an utterance since communication is directly relevant to the current activity. One prediction is thus, that the type of shared activity should influence the communication of caregivers and infants.

Several studies have compared caregivers' speech in different types of activities and have revealed equivocal findings. For example, when comparing activities that involved free play and book reading, Snow et al. (1976) did not find a difference between syntactic aspects in caregivers' language such as the relative frequency of noun phrases and verb phrases. However, Tardif, Gelman, and Xu (1999) found that English-speaking mothers used more verb types than noun types in play situations while the opposite was true in a book reading task. On the macro level, other studies have suggested that caregivers' speech acts differ according to which parent is interacting, the social class of the caregivers, and according to the type of activity (Leaper \& Gleason, 1996; Ryckebusch \& Marcos, 2004; Snow et al., 1976). For example, caregivers use more behavior directives in free play contexts as opposed to book-reading contexts (Hoff-Ginsberg, 1991; Jones \& Adamson, 1987). Little research, however, has addressed the structural differences of the types of shared activities themselves. While the main focus has been on free play tasks that are based on joint manual activities, few studies have considered activities based on mutual regard of objects: an activity argued by Werner and Kaplan (1963) to serve an important role in the understanding of symbols and language learning. Although book-reading may share some of the features of a context of regard, books themselves are objects which infants manipulate, and infants sometimes even attempt to manipulate the objects they depict (DeLoache, Pierroutsakos, Uttal, Rosengren, \& Gottlieb, 1998). Further, in real life situations, the majority of children in non-western cultures are not frequently confronted with book-reading practices and do not learn language usage through book reading but must rely on other formats of mutual object regard.

Deictic gestures are another pivotal aspect in the communication of caregivers and infants. Caregivers frequently use deictic gestures to reinforce the message conveyed in their speech (Iverson, Capirci, Longobardi, \& Caselli, 1999) and infants already use deictic gestures to communicate in meaningful ways before they use language (Bates, Camaioni, \& Volterra, 1975; Liszkowski, in press, for an 
overview). It is currently unknown whether the type of shared activity influences deictic gesture usage. With regard to caregivers, one study suggests that caregivers do not use deictic gestures differently across a free play task (mostly involving book reading) and a counting task (requiring counting several toys together; O’Neill, Bard, Linnell, \& Fluck, 2005). However, in that study, the types of shared activities were not very different from each other, as both involved looking at (pictures of) objects. With regard to infants, no study to date has investigated the influence of the type of shared activity on infants' deictic gesture use. By extending social-pragmatic theories of language acquisition to the gestural modality, we would expect that the usage of non-verbal deictic gestures also varies according to the type of shared activity, for example, if one activity focused on joint manual actions and the other, on joint visual regard of objects. If the type of shared activity influenced infants' use of deictic gestures, this would demonstrate a pervasive influence of shared activities on human communication in the gestural modality from the beginning, even before the emergence of language.

A final aspect relevant to caregiver-infant communication concerns caregivers' multimodal integration of speech and gesture. It is well-established that caregivers integrate gestures with speech (Iverson et al., 1999; O’Neill et al., 2005; Gogate, Bahrick, \& Watson, 2000). For example, Gogate et al. (2000) showed that caregivers 'show' gestures tended to include more movement when caregivers were teaching infants novel verbs as opposed to novel nouns. O’Neill et al. (2005) showed that caregivers' deictic gestures most often disambiguate referents in their speech as opposed to emphasize or add information to the speech. Kalagher and $\mathrm{Yu}$ (2006) further showed that word learning is facilitated when caregivers point to the target referent while naming the object. However, to date, most studies have either focused on a single deictic gesture type (e.g., pointing) or combined all deictic gestures into one category (e.g., deictic gestures). Few studies have investigated whether caregivers systematically combine specific linguistic features with specific forms of deictic gestures, and to what extent different types of shared activities affect how speech and gesture are combined. If the ongoing shared activity influences the use of language and gesture, and if gestures indeed play a facilitative role in the acquisition of language and its usage, we expected that speech and gesture combinations should vary systematically according to the type of shared activity. Caregivers' systematic integration of particular deictic gestures and linguistic features in specific shared activities would provide the beginning language learner with valuable regularities in a multimodal scaffold.

In the current study, we investigated how the type of shared activity shapes caregiver and infant communication and whether caregivers combine particular deictic gestures with specific aspects of speech. We used a new semi-natural context of regard in which caregivers and their twelve-month-old infants spent 
five minutes exploring items displayed around a decorated room. We compared language use (on behalf of caregivers) and deictic gesture use (on behalf of both caregivers and infants) in this new task with language and gesture use in a free play task, in which dyads acted together on objects. Our first goal was to investigate whether different types of activities influenced caregivers' language use with regard to specific speech acts and linguistic references. Our second goal was to determine whether the type of shared activity influenced caregivers' non-verbal, gestural communication and if it might even influence the gestural communication of prelinguistic infants. Our third goal was to determine whether there was a specific pattern with regard to how caregivers combine deictic gestures with language, and to what extent these combinations would be affected by the different types of shared activities. Based on social-pragmatic theories of language acquisition and on previous findings, we expected that the type of shared activity would structure caregiver-infant communication such that caregivers would use both speech and gesture - and infants their gestures - differently in the two contexts. Further, if caregivers combined specific deictic gesture types with specific language features, then we predicted that these combinations too, would differ according to the type of shared activity.

\section{Method}

\section{Participants}

Thirty-nine 12-month-old infants ( mean age $=366$ days; range $=349-398 ; 20$ girls and 19 boys) and their primary caregivers ( 35 mothers and 4 fathers) participated in this study. All of the participants lived in a middle-sized city in Germany; all were native speakers of German, and all spoke German to their infants at home. The participants were the same as those in Liszkowski and Tomasello (submitted).

\section{Procedure}

Caregivers spent approximately five minutes interacting with their infants in two contexts, each of which resembled a different type of shared activity in the daily lives of caregivers and their infants. The first context was the Context of Regard (See Figure 1). The Context of Regard was meant to represent a situation where infants and caregivers spend time together, looking at objects. In order to replicate this social interactional context, a decorated room was designed in which a number of interesting items were placed on the wall and around the room. Some examples of objects in the room included stuffed animals, toys, and several pictures of 


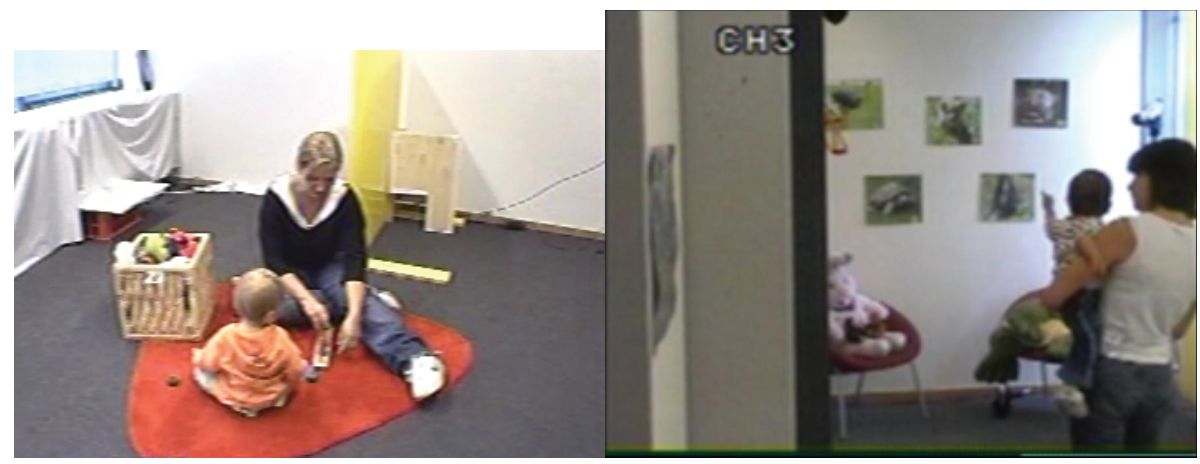

Figure 1. Setup of the two shared activities: Context of Action (left) Context of Regard (right)

animals. Caregivers were instructed to hold their infants on their hips and explore the items in the room with their infants. Participants were all kept blind to the aims of the study and no mention of language or gesture was given in the instructions. In order to assure that the context remained one of regard, caregivers were asked to avoid touching the objects. Each dyad spent approximately five minutes in the room. The entire session was recorded via four video cameras mounted in each of the four corners in the room.

Immediately following the Context of Regard, the participants were asked to participate in the second task: The Context of Action (See Figure 1). The Context of Action was an unstructured free play situation where caregivers spent approximately five minutes interacting with their infants and a box of toys. Caregivers were instructed to interact "as they would do at home." Again, no mention of language or gesture was given in the instructions. The box of toys included a variety of different toys. Some examples include: a ring-stacking game, two toy cars, several stuffed animals, and a set of toy telephones. Four video cameras recorded dyads' interactions.

\section{Coding}

All coding was done in ELAN, a free software program developed by the Max Planck Institute for Psycholinguistics, which allows for coding that is time locked with the video data. The coding focused on three aspects in the interaction: Language, Gestures, and Language \& Gesture combinations.

\section{Language}

All caregiver speech was first transcribed orthographically and time locked with the video data. Since infants produce so little language at 12 months, we only cod- 
ed the language of the caregivers. Specifically, we coded linguistic references and speech acts. For linguistic references, our goal was to code referential words. Thus, we coded all words that were used to refer to objects (e.g., It's a goat), dynamic actions (e.g., He's jumping), and features (e.g., The rabbit is pink). Words that were unclear or incomprehensible were not included in the analysis. Words that were repeated in the same utterance were counted as a single reference. Utterances were defined as any unit of speech preceded and followed by silence (Crystal, 1991). For speech acts, we coded all utterances as Comments (e.g., That's a frog.), Questions (e.g., Where's the fish?), behavioral Directives (e.g., Put it there.), and Invitations (e.g., Look here!). All speech act categories except invitations were adopted from Tomasello and Farrar (1986). Invitations are often used with infants as a summons to draw infants' attention towards objects, after which, caregivers will speak further about the object (Estigarribia \& Clark, 2007). The four speech act categories encompassed all speech such that every utterance was assigned to at least one of the four aforementioned categories. It should also be noted that some utterances were used for more than one speech act. For example, a caregiver might say: "Look, it's a frog." in which case the utterance serves as both an invitation and a comment.

\section{Gestures}

We coded the following deictic gestures of caregivers and infants: Action Demonstration: An individual performs an action with an object, with the intent for the other individual to duplicate the action; Object Demonstration: An individual draws attention to an object by moving and animating it for the other to see; $\underline{\text { Show: }}$ An individual draws attention to an object by holding it in view of the other; Give: An individual transfers possession of an object from self to another; Place: An individual transfers an object to the ground to draw the other's attention to the object; Point: An individual uses the hand or a part of the hand to indicate an external referent to another; Request: An individual requests an object held by another by reaching out one's own hand, palm up; Reach: An individual indicates desire of an object by reaching for it, without the sole intent of retrieving the object by oneself. The gesture categories were based on previous literature and on observation of caregiver-infant interactions (see, e.g., Bates et al., 1975; Blake, O'Rourke, \& Borzellino, 1994; Clark, 2003; Trautman \& Rollins, 2006).

\section{Language \& Gesture combinations}

Language \& Gesture combinations were based on temporal synchrony between the gesture and the speech with which the gesture occurred (Iverson et al., 1999). For linguistic references, the temporal synchrony had to be between the actual referential word and the gesture. For speech acts, the temporal synchrony had to 
be between the utterance in which the speech act occurred and the gesture. An example of a Language \& Gesture combination is provided in (1).

In (1), the point spans across the entire duration of the utterance. The point spans across one speech act (a comment) and two linguistic references (an object reference (rabbit) and an action reference (jumping).

(1) Gesture:

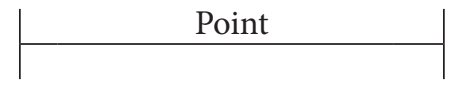

Utterance:

The rabbit is jumping.

Speech act:

Linguistic Reference:

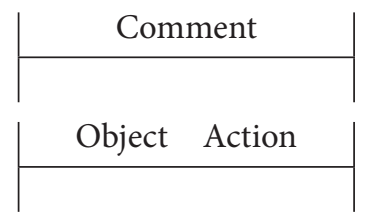

\section{Reliability}

Nine randomly selected dyads were re-coded for language and gesture occurrences by a second, trained assistant. Inter-rater reliability revealed significant correlations for each language category, both with respect to speech acts (all $\rho$ 's $>.86$, $p$ 's $\leq .001$ ), and linguistic references (all $\rho$ 's $>.93$, $p$ 's $\leq .001$ ). For gestures, inter-rater reliability revealed significant correlations for the frequencies of each gesture type $(\rho$ 's $>.69$ p's $<.05){ }^{1}$

\section{Results}

Coding revealed that dyads spent, on average, slightly more time in the Context of Action (mean = 312 seconds) than in the Context of Regard (mean $=301 \mathrm{sec}$ onds), $t(38)=2.409, p=.021$. We therefore calculated language and gesture frequencies per minute, which allowed for comparison between the two activities.

\section{Language}

A 2 (Shared activities) x 3 (Linguistic reference types) repeated measures ANOVA on the mean frequency of references per minute revealed that caregivers used overall significantly more linguistic references in the Context of Regard than in the Context of Action $F(1,38)=23.00, p<.001$. Further, caregivers used linguistic reference types with different frequencies, $F(1.64,62.27)=55.09, p<.001$ (adjusted for Greenhouse-Geisser correction). These two effects interacted significantly, 


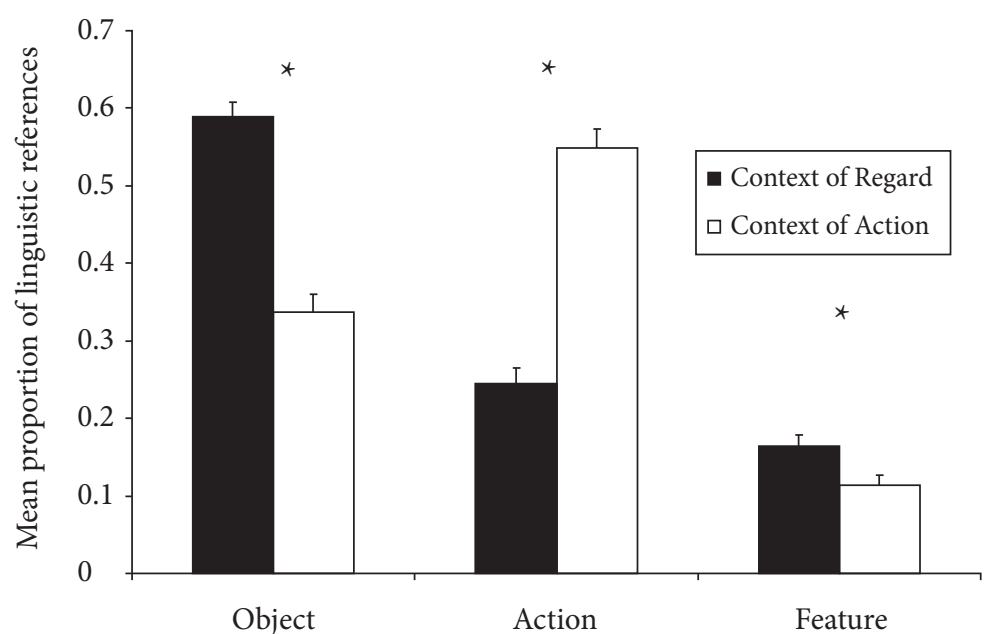

Figure 2. Caregivers' use of linguistic references across contexts

$F(1.63,61.82)=85.84, p<.001$ (adjusted for Greenhouse-Geisser correction). On account of the different amounts of speech in the two activities, we resolved the interaction with $t$-tests on the proportions of linguistic reference types relative to the total amount of linguistic references in each activity. Figure 2 shows that caregivers used significantly more object references and more feature references in the Context of Regard than in the Context of Action, $t(37)=9.36, p<.001, t(37)=2.77$, $p=.009$, respectively. Further, they used significantly more action references in the Context of Action than in the Context of Regard, $t(37)=9.21, p<.001$.

A 2 (Shared activities) x 4 (Speech act types) repeated measures ANOVA on the mean frequency of speech acts revealed that caregivers used significantly more speech acts in the context of Context of Regard than in the Context of Action, $F(1,38)=23.78, p<.001$. Further, caregivers used speech acts with different frequencies, $F(1.41,53.49)=177.80, p<.001$ (adjusted for Greenhouse-Geisser correction). These two effects interacted significantly, $F(2.06,78.19)=18.35$, $p<.001$ (adjusted for Greenhouse-Geisser correction). On account of the different amounts of speech in the two activities, we resolved the interaction with $t$-tests on the proportions of speech act types relative to the total amount of speech acts in each activity. Figure 3 shows caregivers' use of speech acts in the two activities, whereby caregivers used significantly more directives in the Context of Action than in the Context of Regard, $t(37)=6.58, p<.001)$, and more invitations in the Context Regard than in the Context of Action, $t(37)=2.00, p=.052$. There was no significant difference between caregivers' use of comments in the two contexts, $t(37)=.374, p=.71$, nor was there a significant difference in their use of questions, $t(37)=1.70, p=.098$. 


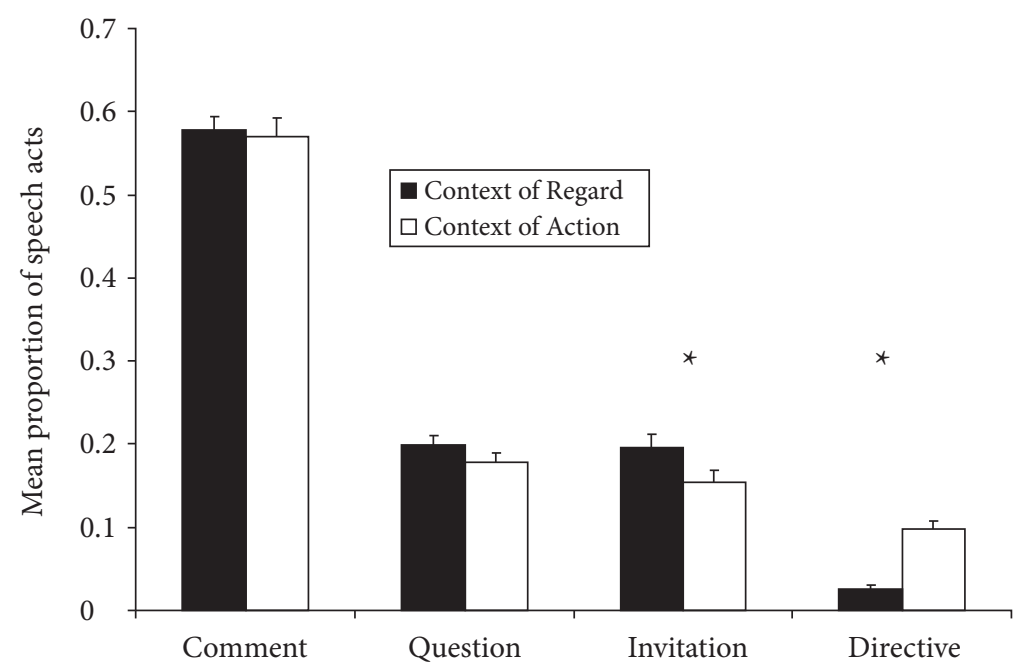

Figure 3. Caregivers' use of speech acts across contexts

\section{Gestures}

Figure 4 shows the frequencies of each gesture type for both caregivers and infants in the Context of Action. As is evident from Figure 4, caregivers and infants used a variety of deictic gestures in the Context of Action. Figure 5 shows that both caregivers and infants pointed significantly more in the Context of Regard than in the Context of Action, $t(38)=6.17, p<.001 ; t(38)=6.60(38) p<.001$, respectively. For caregivers, $92 \%(\mathrm{~N}=36)$ pointed at least once in the Context of Regard, whereas only 59\% ( $\mathrm{N}=23)$ pointed at least once in the Context of Action (McNemar, $p<.001)$. For infants, $95 \%(\mathrm{~N}=37)$ pointed at least once in the Context

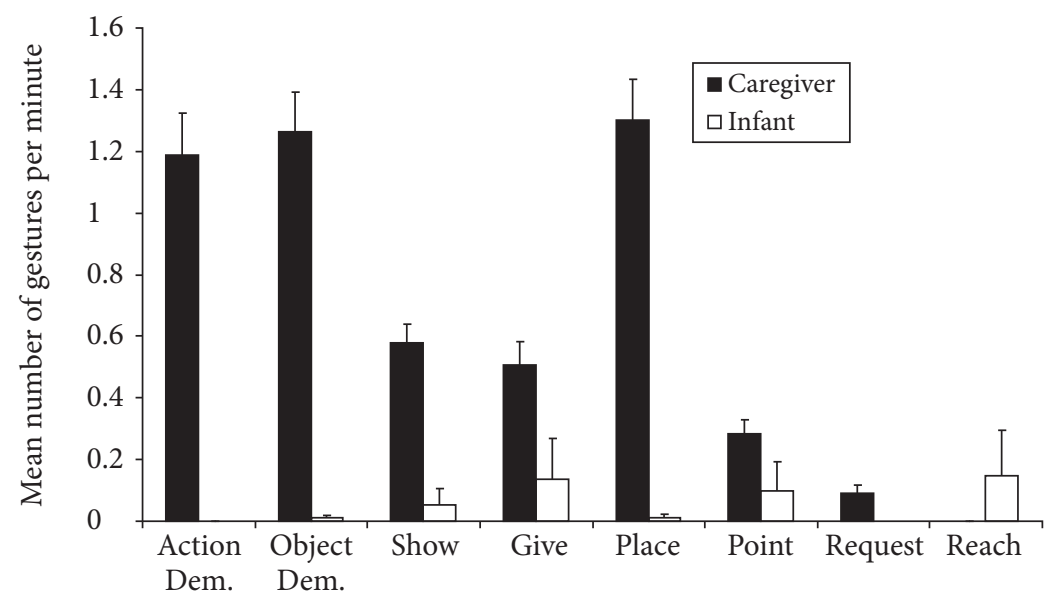

Figure 4. Caregiver and infant gestures in Context of Action 


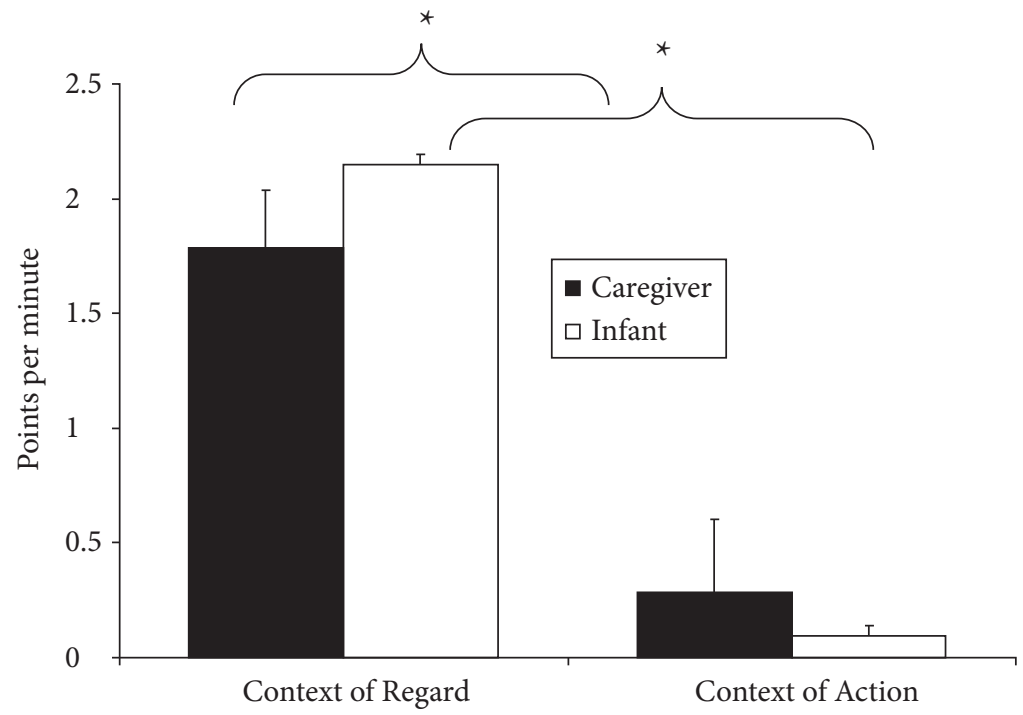

Figure 5. Caregiver and infant pointing across contexts

of Regard, whereas only $23 \%(\mathrm{~N}=9)$ pointed at least once in the Context of Action (McNemar, $p<.001)$.

\section{Language and Gesture combinations}

Figure 6 shows that in both contexts, caregivers' deictic gestures were most often accompanied by speech. In the Context of Action, $63 \%$ of all deictic gestures were

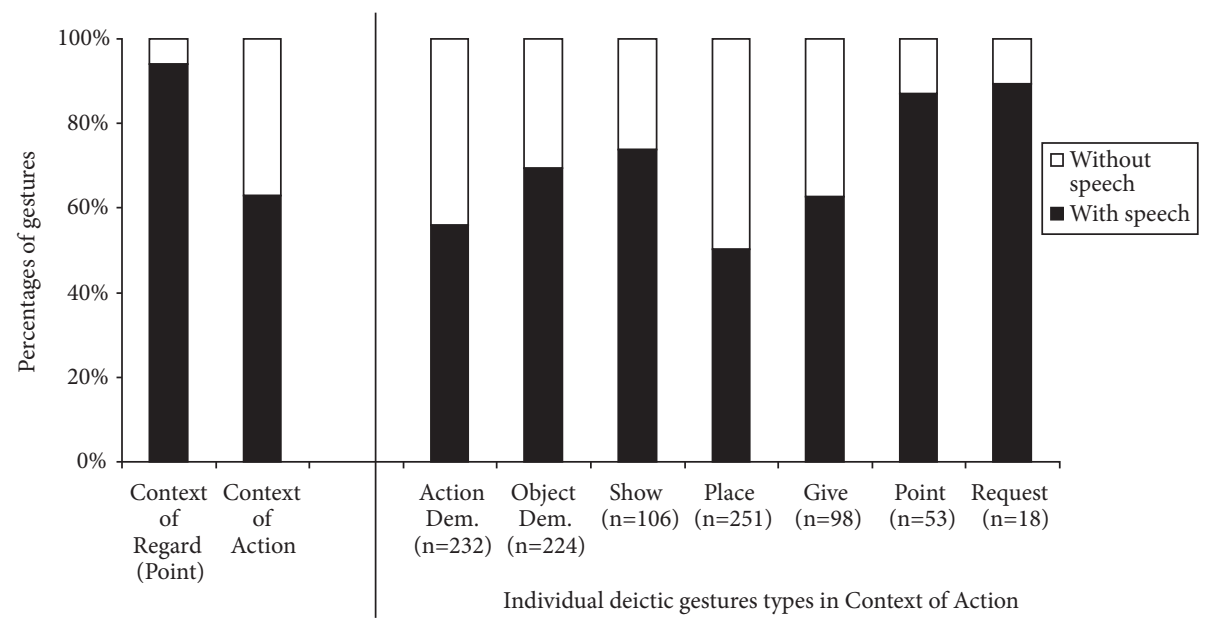

Figure 6. Percentages of caregivers' deictic gestures accompanied by speech across contexts 
accompanied by speech, although there was also variation with respect to each individual gesture type (see Figure 6). In the Context of Regard, 94\% of caregivers' pointing gestures were accompanied by speech.

\section{Context of Action}

Since many of the gestures were used infrequently in the Context of Action and since not all gestures were used by all caregivers, parametric tests were not appropriate. We therefore combined data of all caregivers into one single set and conducted chi-square analyses. The chi-square tests make it possible to discern whether certain gestures co-occur with particular speech act types and linguistic reference types more than would be expected by chance.

A chi-square test revealed that gesture types and linguistic references did not co-occur randomly: $\chi^{2}(12)=66.61, p<.001$. In order to investigate this association further, adjusted standardized residuals (ASRs) were calculated for each individual chi-square cell. ASRs indicate which cells contribute to the significant chi-square value and they allow for the comparison between cells (Agresti, 2007; Sheskin, 2004). These calculations allowed us to determine which particular Gesture \& Speech act and Gesture \& Linguistic reference combinations occurred more or less than would be expected by chance. ASRs above 2.0 reflect a significant association between two variables $(p<.05)$ while ASRs below -2.0 reflect a significant dissociation between two variables ( $p<.05$; Agresti, 2007; Sheskin, 2004). ASRs for the entire chi-square are displayed in Table 1 . The residual analysis revealed significant associations between the following gestures and linguistic references: Action demonstration \& Action reference, Object demonstration \& Object reference, Show \& Object reference, and Give \& Action reference, indicating that

Table 1. Adjusted standardized residuals for Gesture \& Linguistic reference combinations in Context of Action

\begin{tabular}{llll}
\hline & \multicolumn{2}{l}{ Linguistic reference } \\
\cline { 2 - 4 } Gesture & Object & Action & Feature \\
\hline Action demonstration & -4.5 & 4.8 & -0.6 \\
Object demonstration & 5.2 & -3.4 & -2.4 \\
Show & 3.3 & -2.9 & -0.5 \\
Place & -1.1 & -0.3 & 2 \\
Give & -3.5 & 2.1 & 1.9 \\
Point & -0.6 & 0.2 & 0.6 \\
Request & -1.2 & 1.1 & 0.1 \\
\hline
\end{tabular}

Note. Statistically significant associations (positive value) and dissociations (negative value) are in boldface; $p<.05$ 
Table 2. Adjusted standardized residuals for Gesture \& Speech act combinations in Context of Action:

\begin{tabular}{lclcc}
\hline & \multicolumn{2}{l}{ Speech Act } & & \\
\cline { 2 - 5 } Gesture & Comment & Directive & Invitation & Question \\
\hline Action Demonstration & 0.6 & -0.7 & 0.9 & -1.3 \\
Object Demonstration & 2.4 & -1.1 & -1.7 & -0.5 \\
Show & -0.5 & -1.3 & 0.8 & 0.8 \\
Place & -1.5 & -1.1 & 3.1 & -0.5 \\
Give & 0 & 4.1 & -4.4 & 1.7 \\
Point & -1.2 & 2.3 & 1.8 & -2.1 \\
Request & -1.3 & -0.6 & -2.3 & 4.8 \\
\hline
\end{tabular}

Note. Statistically significant associations (positive value) and dissociations (negative value) are in boldface; $p<.05$

these particular Gesture \& Linguistic reference combinations occurred more often than would be expected by chance. There were significant dissociations between the following: Action demonstration \& Object reference, Object demonstration \& Action reference, Object demonstration \& Feature reference, Show \& Action reference, and Give \& Action reference, and indicating that these particular combinations occurred less than would be expected by chance.

Another chi-square analysis was computed on Gesture \& Speech Act combinations in the Context of Action. This revealed that deictic gesture types and speech acts did not co-occur randomly, $\chi^{2}(18)=82.78, p<.001$. Again, ASRs were calculated to further break down the association (see Table 2). These revealed significant associations between: Object demonstration \& Comment; Place \& Invitation; Give \& Directive; Point \& Directive, and Request \& Question, and significant disassociations between: Give \& Invitation; Point \& Question; and Request \& Invitation.

\section{Context of Regard}

In the Context of Regard, we analyzed only the language that accompanied caregivers' points since all of the other gestures involve direct contact with the objects and the participants had been asked not to touch the objects.

Figure 7 shows caregivers' use of Point \& Linguistic reference combinations in the Context of Regard. A one-way repeated measures ANOVA on the mean frequencies of combination types revealed a significant difference between the various Point \& Linguistic reference type combinations in the Context of Regard, $F(1.05)=21.802, p<.001$ (adjusted for Greenhouse Geisser correction). Paired $t$-tests revealed that caregivers used Point $\&$ Object reference combinations significantly more often than Point $\&$ Feature reference combinations, $t(38)=4.815$, 


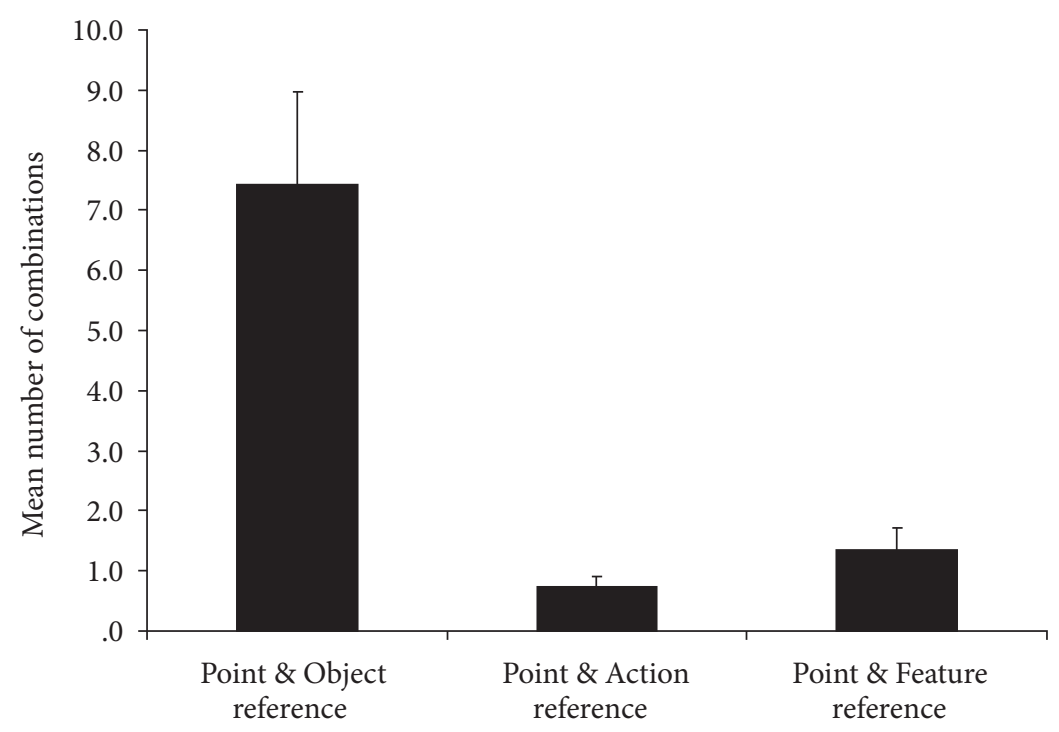

Figure 7. Caregivers' Point \& Linguistic reference combinations in the Context of Regard $p<.001$ and Point \& Action reference combinations $t(38)=4.639, p<.001$. They also significantly used more Point \& Feature references than Point \& Action references, $t(38)=2.037, p=.049$.

Figure 8 shows caregivers' use of Point \& Speech act combinations in the Context of Regard. A one-way repeated measures ANOVA revealed a significant

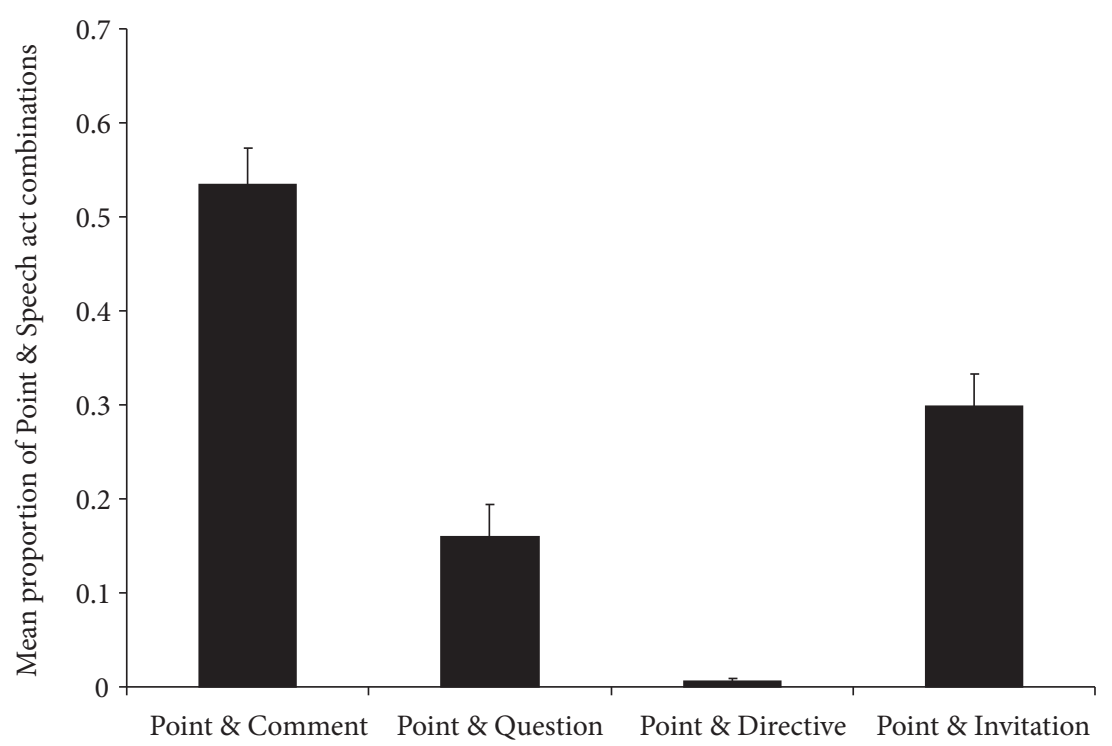

Figure 8. Caregivers' Point $\&$ Speech act combinations in the Context of Regard 
difference between the frequencies of the various combinations, $F(1.37)=26.94$, $p<.001$ (adjusted for Greenhouse-Geisser correction; see Figure 8). To break down this effect, paired $t$-tests were computed. These showed that caregivers used Point \& Comment combinations more than all other combinations (all $p$ 's $<.01$ ), followed by more Point $\&$ Invitation combinations (all $p$ 's $<.01$ ). Point $\&$ Directive combinations were used least often (all p's $<.001$ ).

\section{Discussion}

The current study shows how different types of shared activities structure caregivers' and infants' communication. Depending on the type of shared activity, caregivers exposed infants to different types of linguistic input, to different types of deictic gestures, and to specific gesture-language combinations. The type of shared activity also influenced the way prelinguistic infants communicated with their deictic gestures. The findings support social-pragmatic theories of language usage and acquisition and show how shared activities structure human communication from the beginning. The type of shared activity and caregivers' systematic integration of specific forms of deictic gestures with language provides infants with a multimodal scaffold for a usage-based acquisition of language.

The type of shared activity had a pervasive influence on the verbal and nonverbal communication of caregivers and even on the communication of prelinguistic infants, which reveals that shared activities structure communication irrespective of both the modality and the presence of language. In a context where action and manipulation of objects was possible, both caregivers and their prelinguistic infants used a variety of proximal deictic gestures such as show, place, give, or object demonstration. In this context, they rarely used points. However, when the interaction was focused on regard of objects, both caregivers and infants pointed frequently. It is worth emphasizing that the total amount of objects was approximately equal between the two contexts, thus, infants had equal opportunities to point in each. The high prevalence of the pointing gesture in contexts of regard, and its relative absence in contexts of action suggests that pointing is used in infancy primarily as a means to share interest in distal, non-manipulable objects (Liszkowski, Carpenter, Henning, Striano, \& Tomasello, 2004). In support of this, our speech act analysis revealed that caregivers pointed in the Context of Regard most often to comment, while they pointed in the Context of Action most often to direct infants' behavior. This illustrates how the inherent ambiguity of the pointing gesture (see also Quine, 1960) is disambiguated through the shared activity within which it is used (see also Liebal, Behne, Carpenter, \& Tomasello, 2009). With regard to the ontogenetic origins of pointing, one intriguing 
possibility is that distal reference via pointing may build on an understanding of reference underlying proximal gestures, which may first emerge in the context of shared manual activities.

The influence of the type of shared activity pertained unequivocally to caregivers' language usage. We found more object references when caregivers and infants were in a context based on regard of objects than when they were in a context based on acting on objects. Conversely, there were more action references when dyads were in a context of acting on objects than when they were looking together at objects. This illustrates, on the micro level, how different types of shared activities help narrow the referential interpretation of words. The use of speech acts was also dependent on the type of shared activity: There were more invitations to look at objects when dyads were in the context based on regard of objects than when they were in the context focused on acting on and manipulating objects. Conversely, there were more behavior directives in the Context of Action. On the macro level, this demonstrates how the usage of language is shaped by the type of shared activity.

With regard to the integration of different kinds of deictic gestures and speech, as measured in the Context of Action, we found that caregivers integrated language and gesture systematically: Specific gestures were used in combination with particular types of language. For example, action references systematically accompanied action demonstrations, thus highlighting action, and object references systematically accompanied show gestures, thus highlighting objects. Further, the speech acts that accompany particular gestures offer insight into the various uses of the gestures themselves. Request gestures, for example occurred frequently with questions, e.g., 'Can I have the yellow block?' where the speech and the gesture are both used to request an object from the infant. Although the place gesture and the give gesture are morphologically fairly similar, they were combined with different speech act types, revealing that they are actually used differently from one another. The integration of the two modalities constitutes an advantageous form of reinforcing multimodal communication through which caregivers maintain infants' attention and scaffold their communicative development. It is likely that infants use speech accompanying gestures to narrow down the possible referential interpretations of words. Thus, with the help of activity-dependent, gesture-language combinations, infants themselves can learn to refer by making associations between utterances they hear, the gestures they see, and the objects and actions that are relevant to the ongoing shared activity.

Our results also have important methodological implications. First, the results accentuate the need to consider language acquisition in context: Researchers should be cautious in assuming that a language or gesture sample from a single type of activity (e.g., "free play" in the sense of joint manual action) accurately 
portrays one's communication. They should also be cautious when combining data from multiple settings. Results show that the type of shared activity and caregivers' accompanying gestures play an integral role in shaping caregiver-infant interactions. Researchers studying infants' natural language development should therefore strive for a comprehensive portrayal of infants' language input by including factors other than the linguistic content itself. Second, our findings are also relevant for cross-cultural research. Cultures differ greatly in the amount of time they spend in various types of shared activities, and in how frequently various gesture types are used (Salomo \& Liszkowski, 2010). It is thus likely that infants' social and linguistic development depends on how prevalent certain types of interactions are within the culture.

Taken together, the finding that caregiver and infant communication is shaped by the type of shared activity, and the finding that caregivers integrate language and gesture systematically, lend support to the notion that infants' communicative input is structured by factors other than language itself. These factors help infants infer communicative intent and extract relevance from the input (Bruner, 1975; Tomasello, 2003). Following usage based theories of language acquisition, this enables infants to both disambiguate the intended referent in caregivers' speech, and to learn about the functions for which speech is used. As a whole, our study supports a rich, socio-pragmatic view of language acquisition whereby human communication is structured by non-linguistic gestures and by the activities within which it is used.

\section{Note}

1. Not all correlations for infant gestures were significant. $\rho$ for infants' Reach gestures was .61, $p=.08$, and for infants' Show gesture, $\rho=.55, p=.129$. These correlations are non-significant because infants rarely gestured in this context which greatly reduces the statistical power of the test.

\section{References}

Agresti, Alan (2007). An introduction to categorical data analysis. 2nd ed. Hoboken, NJ: Wiley.

Baldwin, Dare A. (1991). Infants' contribution to the achievement of joint reference. Child Development, 62 (5), 875-890.

Baldwin, Dare A. (1995). Understanding the link between joint attention and language. In C. Moore, P. J. Dunham (Eds.), Joint attention: its origins and role in development (pp.131158) Hillsdale, NJ: Erlbaum.

Bates, Elizabeth, Luigia Camaioni, \& Virginia Volterra (1975). The acquisition of performatives prior to speech. Merrill-Palmer Quarterly, 21 (3), 205-226. 
Blake, Joanna, Paula O'Rourke, \& Grace Borzellino (1994). Form and function in the development of pointing and reaching gestures. Infant Behavior and Development, 17 (2), 195-203.

Bruner, Jerome (1975). The ontogenesis of speech acts. Journal of Child Language, 2 (1), 1-19.

Bruner, Jerome (1981). The social context of language acquisition. Language \& Communication, $1(2 / 3), 155-178$.

Bruner, Jerome (1983). The acquisition of pragmatic commitments. In R. Golinkoff (Ed.), The transition from prelinguistic to linguistic communication (pp. 27-42). Hillsdale, NJ: Erlbaum.

Clark, Herbert (2003). Pointing and placing. In Sotaro Kita (Ed.), Pointing: where language, culture, and cognition meet (pp.243-268). Mahwah, NJ: Erlbaum.

Crystal, David (1991). A dictionary of linguistics and phonetics. 3rd edition. Cambridge, MA: Basil Blackwell.

DeLoache, Judy, Sophia Pierroutsakos, David Uttal, Karl Rosengren, \& Alma Gottlieb (1998). Grasping the nature of pictures. Psychological Science, 9 (3), 205-210.

Estigarribia, Bruno \& Eve Clark (2007). Getting and maintaining attention in talk to young children. Journal of Child Language, 34 (4), 799-814.

Gogate, Lakshmi, Lorraine Bahrick, \& Jilayne Watson (2000). A study of multimodal motherese: The role of temporal synchrony between verbal labels and gestures. Child Development, 71 (4), 878-894.

Hoff-Ginsberg, Erika (1991). Mother-child conversation in different social classes and communicative settings. Child Development, 62 (4), 782-796.

Iverson, Jana, Olga Capirci, Emiddia Longobardi, \& M. Cristina Caselli (1999). Gesturing in mother-child interactions. Cognitive Development, 14, 57-75.

Jones, Celeste \& Lauren Adamson (1987). Language use in mother-child and mother-childsibling interactions. Child Development, 58 (2), 356-366.

Kalagher, Hilary \& Chen Yu (2006). The effects of deictic pointing in word learning. In Proceedings of the 5th International Conference of Development and Learning. Bloomington, IN.

Leaper, Cambell \& Jean Gleason (1996). The relationship of play activity and gender to parent and child sex-typed communication. International Journal of Behavioral Development, 19(4), 689.

Liebal, Kristin, Tanya Behne, Malinda Carpenter, \& Michael Tomasello (2009). Infants use shared experience to interpret pointing gestures. Developmental Science, 12, 264-271.

Liszkowski, Ulf (in press). Deictic and other gestures in infancy. Acción psicológica.

Liszkowski, Ulf, Malinda Carpenter, Anne Henning, Tricia Striano, \& Michael Tomasello (2004). Twelve-month-olds point to share attention and interest. Developmental Science, 7 (3), 297-307.

Liszkowski, Ulf \& Michael Tomasello (submitted). Social, cognitive, and morphological differences in the emergence of pointing at 12 months of age.

Nelson, Katherine (1981). Individual differences in language development: Implications for development and language. Developmental Psychology, 17 (2), 170-187.

O’Neill, Maria, Kim Bard, Maggie Linnell, \& Michael Fluck (2005). Maternal gestures with 20-month-old infants in two contexts. Developmental Science, 8 (4), 352-359.

Quine, Willard vanOrman (1960). Word and object. Cambridge, MA: MIT Press.

Ryckebusch, Céline \& Haydée Marcos (2004). Speech acts, social context and parent-toddler play between the ages of 1;5 and 2;3. Journal of Pragmatics, 36 (5), 883-897.

Salomo, Dorothé \& Ulf Liszkowski (2010). Caretaker-infant social interaction and gestures across cultures. Poster presented at the International Conference on Infant Studies. 
Sheskin, David (2004). Handbook of parametric and nonparametric statistical procedures. CRC Press: USA.

Snow, Catherine, A. Arlman-Rupp, Y. Hassing, J. Jobse, J. Joosten, \& J. Vorster (1976). Mothers' speech in three social classes. Journal of Psycholinguistic Research, 5 (1), 1-20.

Tardif, Twila, Susan Gelman, \& Fan Xu (1999). Putting the "noun bias" in context: a comparison of English and Mandarin. Child Development, 70 (3), 620-635.

Tomasello, Michael (2003). Constructing a language: a usage-based theory of language acquisition. Cambridge, MA: Harvard University Press.

Tomasello, Michael \& Michael Farrar (1986). Joint attention and early language. Child Development, 57 (6), 1454-1463.

Trautman, Carol \& Pamela Rollins (2006). Child-centered behaviors of caregivers with 12-month-old infants: Associations with passive joint engagement and later language. Applied Psycholinguistics, 27 (3), 447-463.

Werner, Heinz \& Bernard Kaplan (1963). Symbol formation: An organismic-developmental approach to language and expression of thought. London: John Wiley \& Sons.

\section{Authors' addresses}

Daniel, Puccini (corresponding author), Mireille Hassemer, Dorothé Salomo, and

Ulf Liszkowski

Max Planck Research Group Communication Before Language

Max Planck Institute for Psycholinguistics

Wundtlaan 1

6525 XD Nijmegen

The Netherlands

daniel.puccini@mpi.nl, mireille.hassemer@mpi.nl, dorothé.salomo@mpi.nl, ulf.liszkowski@ mpi.nl

\section{About the authors}

Daniel Puccini studied linguistics at the University of New Mexico and the University of Amsterdam. He is currently a Ph.D. student in the Communication Before Language Group at the Max Planck Institute for Psycholinguistics in Nijmegen, The Netherlands. His research focuses on gestural communication in infancy.

Mireille Hassemer is a master's student in linguistics at the University of Leipzig, Germany. A portion of the research presented in this article was completed at the Max Planck Institute for Psycholinguistics in the Communication Before Language Group in order to fulfill the requirements for her Master's degree.

Dorothé Salomo is a postdoctoral researcher in the Communication Before Language Group at the Max Planck Institute for Psycholinguistics in Nijmegen, The Netherlands. She conducts crosscultural research on infants' prelinguistic communication and social-cognitive development.

Ulf Liszkowski received his Ph.D. in Psychology from the University of Leipzig, Germany. He is leading the Max Planck Research Group Communication Before Language at the MPI for Psycholinguistics, Nijmegen, The Netherlands. His research addresses infants' prelinguistic communication and their social and cognitive development. 